DOI 10.4171/JEMS/196

Laurent Bartholdi

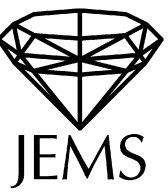

\title{
Gardens of Eden and amenability on cellular automata
}

Received December 10, 2007; revised November 3, 2009

\begin{abstract}
We prove a converse to the "Garden-of-Eden" theorem by Ceccherini-Silberstein, Machì and Scarabotti, and to a theorem by Meyerovitch, yielding two new characterizations of amenable groups. The following are equivalent:

- the group $G$ is amenable;

- all cellular automata living on $G$ that admit mutually erasable patterns also admit gardens of Eden;

- all cellular automata living on $G$ that do not preserve Bernoulli measure admit gardens of Eden.

This solves in particular Conjecture 6.2 (1) in [2].
\end{abstract}

\section{Introduction}

Von Neumann defined ${ }^{1}$ cellular automata as creatures built out of infinitely many finitestate devices arranged on the nodes of $\mathbb{Z}^{2}$ or $\mathbb{Z}^{3}$, each device being capable of interaction with its immediate neighbours. We consider here the natural generalization to creatures living on a graph with simply transitive automorphism group, and show that some fundamental properties of the automaton are characterized by amenability of the underlying graph-a concept also due to von Neumann [15].

Definition 1.1. Let $G$ be a group. A finite cellular automaton on $G$ is a $G$-equivariant continuous map $\Theta: Q^{G} \rightarrow Q^{G}$, where $Q$, the state set, is a finite set.

Note that usually $G$ is infinite; much of the theory holds trivially if $G$ is finite. The map $\Theta$ computes the 1-step evolution of the automaton, and its continuity implies that the evolution of a site depends only on a finite neighbourhood.

For purposes of computation, it is convenient to express a cellular automaton by the following finite amount of data: a finite subset $S$ of $G$, called the memory set, and the restriction $\theta: Q^{S} \rightarrow Q^{\{1\}}$ of $\Theta$. The original cellular automaton is then recovered by setting

$$
\Theta(\phi)(x)=\theta(s \mapsto \phi(x s))
$$

for all $\phi: G \rightarrow Q$, which are called configurations.

L. Bartholdi: Mathematisches Institut, Georg-August Universität zu Göttingen, Bunsenstraße 3-5, D-37073 Göttingen, Germany; e-mail: laurent.bartholdi@gmail.com

1 It seems that von Neumann never published his work on cellular automata—see [1] for history of the subject. 
Note that $S$ may be supposed to generate $G$, although this is by no means a necessity. In general, if $\langle S\rangle=H \leq G$, then the evolution of the automaton is that of $G / H$ parallel, independent cellular automata on $H$.

A cellular automaton should be thought of as a highly regular animal, composed of many cells labelled by $G$, each in a state $\in Q$. Each cell "sees" its neighbours as defined by $S$, and "evolves" according to its neighbours' states.

Two properties of cellular automata received special attention. A pattern is the restriction of a configuration to a finite subset $Y \subseteq G$. On the one hand, there can exist patterns that never appear in the image of $\Theta$. These are called Gardens of Eden (GOE), the biblical metaphor expressing the notion of paradise lost forever.

On the other hand, $\Theta$ can be non-injective in a strong sense: there can exist patterns $\phi_{1}^{\prime} \neq \phi_{2}^{\prime} \in Q^{Y}$ such that, however one extends $\phi_{1}^{\prime}$ to a configuration $\phi_{1}$, if one extends $\phi_{2}^{\prime}$ similarly (i.e. in such a way that $\phi_{1}$ and $\phi_{2}$ have the same restriction to $G \backslash Y$ ) then $\Theta\left(\phi_{1}\right)=\Theta\left(\phi_{2}\right)$. These patterns $\phi_{1}^{\prime}, \phi_{2}^{\prime}$ are called Mutually Erasable Patterns (MEP). Equivalently ${ }^{2}$ there are two configurations $\phi_{1}, \phi_{2}$ which differ on a non-empty finite set, with $\Theta\left(\phi_{1}\right)=\Theta\left(\phi_{2}\right)$. The absence of MEP is sometimes called pre-injectivity [7, §8.G].

Cellular automata were initially considered on $G=\mathbb{Z}^{n}$. Celebrated theorems by Moore and Myhill [13, 14] prove that, in this context, a cellular automaton admits GOE if and only if it admits MEP; necessity is due to Myhill, and sufficiency to Moore. This result was generalized by Machì and Mignosi [10] to groups of subexponential growth, and by Ceccherini-Silberstein, Machì and Scarabotti [2] to amenable groups.

There is a natural measure, the Bernoulli measure, on the configuration space $Q^{G}$ : it assigns measure $1 / \# Q$ to each of the clopen sets $\mathcal{U}_{x, q}=\left\{\phi \in Q^{G}: \phi(x)=q\right\}$. Note that the action of $G$ by translation preserves this measure. Hedlund proved (see [8. Theorem 5.4] or [4, Corollary 2.3]), for $G=\mathbb{Z}$, that a cellular automaton preserves Bernoulli measure if and only if it has no GOE. This result was generalized by Meyerovitch [11, Proposition 5.1] to amenable groups.

We prove that these last two results are essentially optimal, and yield new characterizations of amenable groups:

Theorem 1.2. Let $G$ be a group. Then the following are equivalent:

(1) the group $G$ is amenable;

(2) all cellular automata on $G$ that admit MEP also admit GOE;

(3) all cellular automata on $G$ that do not preserve Bernoulli measure admit GOE.

Schupp had already asked in [16, Question 1] in which precise class of groups the MooreMyhill theorem holds. Ceccherini-Silberstein et al. write in [2] $]^{3}$

Conjecture 1.3 ([2, Conjecture 6.2]). Let $G$ be a non-amenable finitely generated group. Then for any finite and symmetric generating set $S$ of $G$ there exist cellular automata $\Theta_{1}, \Theta_{2}$ with that $S$ such that

\footnotetext{
2 In the non-trivial direction, let $\phi_{1}, \phi_{2}$ differ on a non-empty finite set $F$; set $Y=F\left(S \cup S^{-1}\right)$ and let $\phi_{1}^{\prime}, \phi_{2}^{\prime}$ be the restrictions of $\phi_{1}, \phi_{2}$ to $Y$ respectively.

3 I changed their wording slightly to match this paper's.
} 
(1) in $\Theta_{1}$ there are $M E P$ but no GOE;

(2) in $\Theta_{2}$ there are GOE but no MEP.

As a first step, we will prove Theorem 1.2, in which we allow ourselves to choose an appropriate subset $S$ of $G$. Next, we extend a little the construction to answer the first part of Conjecture 1.3 .

Theorem 1.4. Let $G=\langle S\rangle$ be a finitely generated, non-amenable group. Then there exists a cellular automaton $\Theta: Q^{G} \rightarrow Q^{G}$ with memory set $S$ that has MEP but no GOE. Furthermore, this automaton does not preserve Bernoulli measure.

We conclude that the property of "satisfying Moore's theorem", or "satisfying Hedlund's theorem", is independent of the memory set (provided that it generates a non-amenable subgroup), a fact which was not obvious a priori.

Note that Conjecture 1.3 was already known to hold for groups with a non-abelian free subgroup (see [2, Theorem 6.1]).

\section{Proof of Theorem 1.2}

The implication $(1) \Rightarrow(2)$ has been proven by Ceccherini-Silberstein et al.; see also [7, $\S 8]$ for a slicker proof. The implication $(3) \Rightarrow(2)$ holds for all groups, because Bernoulli measure has full support. The implication (2) $\Rightarrow(3)$ is [11, Proposition 5.1]. We need only prove $(2) \Rightarrow(1)$.

Let us therefore be given a non-amenable group $G$. Let us also, as a first step, be given a large enough finite subset $S$ of $G$. Then there exists a "bounded propagation $2: 1$ compressing vector field" on $G$ : a map $f: G \rightarrow G$ such that $f(x)^{-1} x \in S$ and $\# f^{-1}(x)=2$ for all $x \in G$.

We construct the following automaton $\theta$. Its state set is

$$
Q=S \times\{0,1\} \times S .
$$

Order $S$ in an arbitrary manner, and choose an arbitrary $q_{0} \in Q$. Define $\theta: Q^{S} \rightarrow Q$ as follows:

$$
\theta(\phi)= \begin{cases}(p, \alpha, q) & \text { if there exist unique } s<t \text { in } S \text { with }\left\{\begin{array}{l}
\phi(s)=(s, \alpha, p), \\
\phi(t)=(t, \beta, q),
\end{array}\right. \\
q_{0} & \text { if no such } s, t \text { exist, or if too many exist. }\end{cases}
$$

\section{1. $\Theta$ is surjective}

That is, $\theta$ does not admit GOE. Let indeed $\phi$ be any configuration. We construct a configuration $\psi$ with $\Theta(\psi)=\phi$.

Consider in turn all $x \in G$; write $\phi(x)=(p, \alpha, q)$, and $f^{-1}(x)=\{x s, x t\}$ for some $s, t \in S$ with $s<t$. Set then

$$
\psi(x s)=(s, \alpha, p), \quad \psi(x t)=(t, 0, q) .
$$


Note that $\psi(z)=\left(f(z)^{-1} z, *, *\right)$ for all $z \in G$. Since $\# f^{-1}(z)=2$ for all $z \in G$, it is clear that, for every $x \in G$, there are exactly two $s \in S$ such that $\psi(x s)=(s, *, *)$; call them $s, t$, ordered so that $\psi(x s)=(s, \alpha, p)$ and $\psi(x t)=(t, 0, q)$. Then $\Theta(\psi)(x)=$ $(p, \alpha, q)$, so $\Theta(\psi)=\phi$.

\section{2. $\Theta$ is not pre-injective}

That is, $\theta$ admits MEP. Let indeed $\phi: G \rightarrow Q$ be any configuration; then construct $\psi$ following 2.2), and define $\psi^{\prime}$ as follows. Choose any $y \in G$, write $\phi(y)=(p, \alpha, q)$, and write $f^{-1}(y)=\{y s, y t\}$ for some $s, t \in S$ with $s<t$. Define $\psi^{\prime}: G \rightarrow Q$ by

$$
\psi^{\prime}(x)= \begin{cases}\psi(x) & \text { if } x \neq y t \\ (t, 1, q) & \text { if } x=y t\end{cases}
$$

Then $\psi$ and $\psi^{\prime}$ differ only at $y t$; and $\Theta(\psi)=\Theta\left(\psi^{\prime}\right)$ because the value of $\beta$ is unused in 2.1. We conclude that $\theta$ has MEP.

\section{3. $\Theta$ does not preserve Bernoulli measure}

Consider the open set

$$
A=\left\{\phi \in Q^{G}: \phi(1)=q_{0}\right\} .
$$

Let $\mu$ denote Bernoulli measure; then $\mu(A)=1 / \# Q$. Write $Q^{G}=X \sqcup X^{\prime}$, where

$$
X=\{\phi: \text { there are exactly two } s \in S \text { such that } \phi(s)=(s, *, *)\}
$$

and $X^{\prime}=Q^{G} \backslash X$. Clearly $\mu(X), \mu\left(X^{\prime}\right)>0$. Consider $B=\Theta^{-1}(A)$. Then $X^{\prime} \subseteq B$, and $\mu(B \cap X) / \mu(X)=1 / \# Q$ because the restriction of the local rule to $X$ is invariant under any permutation of $Q$. We get

$$
\mu(B)=\mu(B \cap X)+\mu\left(B \cap X^{\prime}\right)=\mu(X) / \# Q+\mu\left(X^{\prime}\right)>1 / \# Q=\mu(A) .
$$

\section{Proof of Theorem 1.4}

We begin by a slightly extended formulation of amenability for finitely generated groups:

Lemma 3.1. Let $G$ be a finitely generated group. The following are equivalent:

(1) the group $G$ is not amenable;

(2) for every generating set $S$ of $G$, there exist $m>n \in \mathbb{N}$ and an " $m: n$ compressing correspondence on $G$ with propagation $S$ ”, i.e. a function $f: G \times G \rightarrow \mathbb{N}$ such that

$$
\forall y \in G: \quad \sum_{x \in G} f(x, y)=m,
$$




$$
\begin{aligned}
\forall x \in G: & \sum_{y \in G} f(x, y)=n, \\
\forall x, y \in G: & f(x, y) \neq 0 \Rightarrow x \in y S .
\end{aligned}
$$

Note that this definition generalizes the notion of " $2: 1$ compressing vector field" introduced above. Indeed, $f$ could be thought of as a multivalued function, which at $x$ takes $f(x, y)$ times the value $y$; we write $f(x)=\{y: f(x, y)>0\}$ and $f^{-1}(y)=\{x$ : $f(x, y)>0\}$.

Proof. For the forward direction, assuming that $G$ is non-amenable, there exists a rational $m / n>1$ such that every finite $F \subseteq G$ satisfies

$$
\#(F S) \geq(m / n) \# F .
$$

Construct the following bipartite oriented graph: its vertex set is $G \times\{1, \ldots, m\} \sqcup G \times$ $\{-1, \ldots,-n\}$. There is an edge from $(g, i)$ to $(g s,-j)$ for all $s \in S$ and all $i \in$ $\{1, \ldots, m\}, j \in\{1, \ldots, n\}$. By hypothesis, every finite $F \subseteq G \times\{1, \ldots, m\}$ has at least $\# F$ neighbours. Since $m>n$ and multiplication by a generator is a bijection, every finite $F \subseteq G \times\{-1, \ldots,-n\}$ also has at least $\# F$ neighbours.

We now invoke the Hall-Rado theorem [12]: if a bipartite graph is such that every subset of any of the parts has as many neighbours as its cardinality, then there exists a "perfect matching"-a subset $I$ of the edge set of the graph such that every vertex is contained in precisely one edge in $I$. Set then

$$
\begin{aligned}
& f(x, y)=\#\{(i, j) \in\{1, \ldots, m\} \times\{1, \ldots, n\}: \\
&I \text { contains the edge between }(x,-j) \text { and }(y, i)\} .
\end{aligned}
$$

For the backward direction: assume that $G$ is amenable, and let $f$ be a boundedpropagation $m: n$ compressing correspondence. Let $S$ be a finite set such that $y^{-1} x \in S$ whenever $f(x, y) \neq 0$, and let $F \subset G$ be a finite set such that $\#(F S)<(m / n) \# F$, a Følner set. Then $y \in F$ and $f(x, y) \neq 0$ imply $x \in F S$, so

$$
m \# F=\sum_{y \in F} \sum_{x \in G} f(x, y) \leq \sum_{x \in F S} \sum_{y \in G} f(x, y)=n \#(F S),
$$

a contradiction.

Let now $G=\langle S\rangle$ be a non-amenable group, and apply Lemma 3.1 to $G=\langle S\rangle$, yielding $m>n \in \mathbb{N}$ and a contracting $m: n$ correspondence $f$. Consider the following cellular automaton $\theta$ with state set

$$
Q=\left(S \times\{0,1\} \times S^{n}\right)^{n} .
$$

Choose $q_{0} \in Q$, and give a total ordering to $S \times\{1, \ldots, n\}$.

Consider $\phi \in Q^{S}$. To define $\theta(\phi)$, seek whether there exists a unique sequence $\left(s_{1}, k_{1}\right)<\cdots<\left(s_{m}, k_{m}\right)$ in $(S \times\{1, \ldots, n\})^{m}$ such that

$$
\phi\left(s_{j}\right)_{k_{j}}=\left(s_{j}, \alpha_{j}, t_{j, 1}, \ldots, t_{j, n}\right) \in S \times\{0,1\} \times S^{n} \quad \text { for } j=1, \ldots, m .
$$


If there are no, or too many, such $s_{1}, k_{1}, \ldots, s_{m}, k_{m}$, set $\theta(\phi)=q_{0}$; otherwise, set

$$
\theta(\phi)=\left(\left(t_{1,1}, \alpha_{1}, t_{2,1}, \ldots, t_{n+1,1}\right), \ldots,\left(t_{1, n}, \alpha_{n}, t_{2, n}, \ldots, t_{n+1, n}\right)\right) \in Q .
$$

The same arguments as before apply. Given $\phi: G \rightarrow Q$, we construct $\psi: G \rightarrow Q$ such that $\Theta(\psi)=\phi$, as follows. We think of the coordinates $\psi(x)_{k}$ of $\psi(x)$ as $n$ "slots", initially all "free", and will use the $m: n$ correspondence $f$ to establish a correspondence between the slots of $\phi$ and those of $\psi$.

By definition, $\# f^{-1}(x)=m$ for all $x \in G$, while $\# f(x)=n$. Consider in turn all $x \in G$; write $f^{-1}(x)=\left\{x s_{1}, \ldots, x s_{m}\right\}$, and let $k_{1}, \ldots, k_{m} \in\{1, \ldots, n\}$ be "free" slots in $\psi\left(x s_{1}\right), \ldots, \psi\left(x s_{m}\right)$ respectively. By the definition of $f$, there always exist sufficiently many free slots.

Mark now these slots as "occupied". Reindex $s_{1}, k_{1}, \ldots, s_{m}, k_{m}$ in such a way that $\left(s_{1}, k_{1}, \ldots, s_{m}, k_{m}\right)$ is minimal among its $m$ ! permutations. Set then

$$
\psi\left(x s_{j}\right)_{k_{j}}=\left(s_{j}, \alpha_{j}, t_{j, 1}, \ldots, t_{j, n}\right) \quad \text { for } j=1, \ldots, m,
$$

where $\alpha_{n+1}, \ldots, \alpha_{m}$ are taken to be arbitrary values (say 0 for definiteness) and

$$
\phi(x)=\left(\left(t_{1,1}, \alpha_{1}, t_{2,1}, \ldots, t_{n+1,1}\right), \ldots,\left(t_{1, n}, \alpha_{n}, t_{2, n}, \ldots, t_{n+1, n}\right)\right) .
$$

Finally, define $\psi$ arbitrarily on slots that are still "free".

It is clear that $\Theta(\psi)=\phi$, so $\theta$ does not have GOE. On the other hand, $\theta$ has MEP as before, because the values of $\alpha_{j}$ in (3.4) are not used for $j \in\{n+1, \ldots, m\}$.

Similarly, setting $A=\left\{\phi \in Q^{G}: \phi(1)=q_{0}\right\}$, we have $\mu\left(\Theta^{-1}(A)\right)>\mu(A)$ as before.

\section{Remarks}

\section{1. $G$-sets}

A cellular automaton could more generally be defined on a right $G$-set $X$. There is a natural notion of amenability for $G$-sets, but it is not clear exactly to what extent Theorem 1.2 can be generalized to that setting — certainly not verbatim, since the $G$-set $G \sqcup\{\cdot\}$ is amenable for all $G$, but may support automata with MEP but without GOE. It is also unclear how to construct automata on graphs with a transitive, but not simply transitive, automorphism group (see e.g. [5]).

\subsection{Myhill's theorem}

It seems harder to produce counterexamples to Myhill's theorem (“GOE imply MEP”) for arbitrary non-amenable groups, although there exists an example on $C=\mathbb{Z} / 2 * \mathbb{Z} / 2 * \mathbb{Z} / 2$, due to Muller ${ }^{4}$ Let us make our task even harder, and restrict ourselves to linear automata over finite rings (so we assume $Q$ is a module over a finite ring and the map $\Theta: Q^{G} \rightarrow$ $Q^{G}$ is linear). The following approach seems promising.

\footnotetext{
4 In his University of Illinois 1976 class notes, see [10, p. 55].
} 
Conjecture 4.1 (Folklore? I learnt it from V. Guba). Let G be a group. The following are equivalent:

(1) The group $G$ is amenable.

(2) Let $\mathbb{K}$ be a field. Then $\mathbb{K} G$ admits right common multiples, i.e. for any $\alpha, \beta \in \mathbb{K} G$ there exist $\gamma, \delta \in \mathbb{K} G$ with $\alpha \gamma=\beta \delta$ and $(\gamma, \delta) \neq(0,0)$.

This last condition, if $\mathbb{K} G$ is a domain, is equivalent to Ore's condition, implying the existence of a classical ring of fractions-see [9] and [6]. The following direction is classical:

Proof of Conjecture 4.1 (1) $\Rightarrow(2)$. Assume that $G$ is amenable, and let $\alpha, \beta \in \mathbb{K} G$ be given. Let $S \subseteq G$ be a finite set containing the supports of $\alpha$ and $\beta$. By Følner's criterion, there exists $F \subseteq G$ finite such that $\#(S F)<2 \# F$. Consider $\gamma, \delta \in \mathbb{K} F$ as variables; then the equation system $\alpha \gamma=\beta \delta$ is linear, has $2 \# F$ unknowns, and at most \#(SF) equations, so has a non-trivial solution.

Conjecture 4.2 (A possible converse to Myhill's Theorem). Let $\mathbb{K}$ be a field. The following are equivalent:

(1) The group $G$ is amenable.

(2) Any $\mathbb{K}$-linear cellular automaton which admits gardens of Eden also admits mutually erasable patterns.

Proof, assuming Conjecture 4.1 Ceccherini-Silberstein and Coornaert proved the (1) $\Rightarrow$ (2) direction in [3, Theorem 1.2].

Assume now the "hard" direction of Conjecture 4.1. Given $G$ non-amenable, we may then find a finite field $\mathbb{K}$, and $\alpha, \beta \in \mathbb{K} G$ that do not have a common right multiple.

Set $Q=\mathbb{K}^{2}$ with basis $\left(e_{1}, e_{2}\right)$, let $S$ contain the inverses of the supports of $\alpha$ and $\beta$, and define the cellular automaton $\theta: Q^{S} \rightarrow Q$ by

$$
\theta(\phi)=\sum_{x \in G}\left(\alpha\left(x^{-1}\right)\left\langle\phi(x) \mid e_{1}\right\rangle-\beta\left(x^{-1}\right)\left\langle\phi(x) \mid e_{2}\right\rangle, 0\right) .
$$

Then $\theta$ has GOE, indeed any configuration not in $(\mathbb{K} \times\{0\})^{G}$ is a GOE. On the other hand, assume for contradiction that $\theta$ had MEP; then by linearity we might as well assume $\Theta(\phi)=0$ for some non-zero finitely-supported $\phi: G \rightarrow Q$. Write $\phi=(\gamma, \delta)$ in coordinates; then $\Theta(\phi)=0$ would give $\alpha \gamma=\beta \delta$, showing that $\alpha, \beta$ actually did have a common right multiple.

Muller's example is in fact a special case of this construction, with

$$
G=\left\langle x, y, z \mid x^{2}, y^{2}, z^{2}\right\rangle,
$$

$\mathbb{K}=\mathbb{F}_{2}$, and $\alpha=x, \beta=y+z$.

Acknowledgments. I am indebted to Nicolas Monod for conversations and explanations on amenability, especially regarding Lemma 3.1 to Tom Meyerovitch for pointing out to me the connection between his work and this paper's construction, and to Peter Keevash, Fabrice Krieger and Andreas Thom for helpful discussions.

This work was partially supported by a CNRS visiting position at Université de Provence, Marseille. 


\section{References}

[1] Burks, A. W. (ed.): Essays on Cellular Automata. Univ. of Illinois Press, Urbana, IL (1970) Zbl 0228.94013 MR 0299409

[2] Ceccherini-Silberstein, T. G., Machì, A., Scarabotti, F.: Amenable groups and cellular automata. Ann. Inst. Fourier (Grenoble) 49, 673-685 (1999) Zbl 0920.43001 MR 1697376

[3] Ceccherini-Silberstein, T., Coornaert, M.: The Garden of Eden theorem for linear cellular automata. Ergodic Theory Dynam. Systems 26, 53-68 (2006) Zbl 1085.37008 MR 2201937

[4] Coven, E. M., Paul, M. E.: Endomorphisms of irreducible subshifts of finite type. Math. Systems Theory 8, 167-175 (1974/75) Zbl 0309.54032 MR 0383378

[5] Diestel, R., Leader, I.: A conjecture concerning a limit of non-Cayley graphs. J. Algebraic Combin. 14, 17-25 (2001) Zbl 0985.05020 MR 1856226

[6] Elek, G.: The amenability and non-amenability of skew fields. Proc. Amer. Math. Soc. 134, 637-644 (2006) Zbl 1152.12002 MR 2180879

[7] Gromov, M.: Endomorphisms of symbolic algebraic varieties. J. Eur. Math. Soc. 1, 109-197 (1999) Zbl 0998.14001 MR 1694588

[8] Hedlund, G. A.: Endormorphisms and automorphisms of the shift dynamical system. Math. Systems Theory 3, 320-275 (1969) Zbl 0182.56901 MR 0259881

[9] Kirillov, A. A., Kontsevich, M. L., Molev, A. I.: Algebras of intermediate growth. Akad. Nauk SSSR Inst. Prikl. Mat. Preprint 39 (1983), 19 pp. (in Russian); English transl. Selecta Math. Soviet. 9, no. 2, 137-153 (1990) Zbl 0705.17013 MR 753873

[10] Machì, A., Mignosi, F.: Garden of Eden configurations for cellular automata on Cayley graphs of groups. SIAM J. Discrete Math. 6, 44-56 (1993) Zbl 0768.68103 MR 1201989

[11] Meyerovitch, T.: Finite entropy for multidimensional cellular automata. Ergodic Theory Dynam. Systems 28, 1243-1260 (2008) Zbl 1152.37009 MR 2437229

[12] Mirsky, L.: Transversal Theory. An Account of Some Aspects of Combinatorial Mathematics. Math. Sci. Engrg. 75, Academic Press, New York (1971) Zbl 0282.05001 MR 0282853

[13] Moore, E. F., Machine models of self-reproduction. In: Mathematical Problems in the Biological Sciences Proc. Sympos. Appl. Math. 14, Amer. Math. Soc., 17-32 (1962) Zbl 0126.32408 MR 0299409

[14] Myhill, J.: The converse of Moore's Garden-of-Eden theorem. Proc. Amer. Math. Soc. 14, 685-686 (1963) Zbl 0126.32501 MR 0155764

[15] von Neumann, J.: Zur allgemeinen Theorie des Masses. Fund. Math. 13, 73-116 and 333 $(1929)=$ Collected Works, Vol. I, 599-643 Zbl 55.0151.01

[16] Schupp, P. E.: Arrays, automata and groups-some interconnections. In: Automata Networks (Argelès-Village, 1986), Lecture Notes in Comput. Sci. 316, Springer, Berlin, 19-28 (1988) Zbl 0658.68057 MR 961274 